\title{
COMPARISON OF ANALYTIC HIERARCHY PROCESS AND INFORMATION AXIOM FOR DESIGN EVALUATION
}

\author{
Mitchell M. Tseng and Xianyun Wang \\ Department of Industrial Engineering and Engineering Management \\ Hong Kong University of Science and Technology \\ Clear Water Bay, Hong Kong \\ tseng@ust.hk/iewxy@ust.hk
}

\begin{abstract}
Design evaluation is often considered as a decision-making problem. Assessing competing alternatives with a given set of criteria is usually a very challenging step in the design process. In the axiomatic design theory, according to the Information Axiom (IA), design options are evaluated on their information content, which reflects the probability of a design alternative successfully satisfying multiple design criteria. Following the design hierarchy and concerns in the axiomatic design theory, this paper proposes a new Analytic Hierarchy Process (AHP) approach to design alternative evaluation, wherein an objective and globally consistent priority measurement is used. It proves that in cases that all criteria are equally important, both IA and AHP methods lead to identical results. Taking the relative importance of the criteria into consideration, however, the results are different. Finally, some general remarks about using these approaches to evaluate design alternatives are highlighted.
\end{abstract}

\section{Introduction}

The design process can be viewed as a mapping process from a set of customer's needs and wants to a set of Functional Requirements (FRs) in the functional domain and then to the corresponding Design Parameters (DPs) in the physical domain (Suh, 1990). Usually, there can be many designs that are equally acceptable from the functional point of view. However, one of these designs may be superior to others in terms of the probability of success in achieving the design goals as expressed by the FRs (Suh, 1990; Suh, 1998). Therefore, in the axiomatic design theory, the success probability is transformed into information content and adopted to evaluate the potential design alternatives. This is one of the methods to select the best design among those competing alternatives evaluated under different, even conflicting criteria (Jiao \& Tseng 1998).

In general, design evaluation is a multicriteria decision-making problem. The Analytic Hierarchy Process (AHP), being one of the most influential methods in multicriteria decision making (Vargas \& Zahedi, 1993), provided us with a comprehensive framework for solving such problems. In a relatively short period of time, about twenty years since Thomas L. Saaty first published the book on AHP (Saaty, 1980), it has been extensively analyzed, applied to numerous and diverse practical problems, and incorporated in many decision making models. However, the hierarchy construction and the priority assessment are always a headache for the applications. Incorporating the domain mapping process and the Information Axiom (IA) in Axiomatic Design Theory, this paper attempts to achieve two main objectives: 1) to propose a new AHP-based approach in design evaluation; and 2) draw some insights by comparing the new approach to the conventional IA-based approach. Pursuing a higher probability of satisfying FRs is the underlying common starting point of the two approaches. 


\section{Background Review}

The axiomatic design theory has attracted much attention from the academia and the industry since.the first book on this subject, The Principle of Design, was published in 1990. Based on the Independence Axiom and the Information Axiom, the axiomatic design process is developed as a systematic approach to design which is often been considered as an art. The former one states that the independence of FRs must always be maintained. The latter states that among those designs that satisfy the Independence Axiom, the design that has the smallest information content is the best design.

Accordingly the information content $I$ is defined in terms of the probability of a set of design settings, often called as DPs in the axiomatic design theory, satisfying a given FR. Suppose the probability of success of a DP satisfying a given FR is $p$, the information content $I$ associated with the probability is defined as $I=\log _{2} \frac{1}{p}=-\log _{2} p$. The information is given in units of bits. The logarithmic function is chosen so that the information content will be additive when there are many functional requirements that must be simultaneously satisfied.

In the general case of $n$ FRs (indexed by $i)$ for an uncoupled design (accordingly, there are $n$ DPs), suppose there are $m$ design alternatives (indexed by $j), I_{j}$ may be expressed as

$$
I_{j}=\sum_{i=1}^{n} \log _{2} \frac{1}{p_{i j}}=-\sum_{i=1}^{n} \log _{2} p_{i j}
$$

where, $I_{j}^{* *}$ is the information content of the $j$ th design alternative;

$p_{i j}$ is the probability of $\mathrm{DP}_{\mathrm{i}}$ of the $j$ th design alternative satisfying $\mathrm{FR}_{\mathbf{i}}$.

In the case of the system range following the uniform distribution, Eq. (1) can be also expressed as

$$
I_{j}=\sum_{i=1}^{n} \log _{2} \frac{S R_{i j}}{C R_{y j}}
$$

Where, $S R_{l j}$ is the system range of $D P_{l}$ of the $j$ th design alternative; $C R_{l j}$ is the common range under the system range of $D P_{i}$ of the $j$ th design alternative and the design range of $F R_{i}$ (Suh, 1998).

Based on the Information Axiom, the design alternative $j$ with the minimum $I_{j}$ will be chosen as the best design in terms of the probability of satisfying the FRs in the design evaluation process.

What decision-makers need is not a more complicated way of thinking notwithstanding the world is a complex system of interacting elements, since it is difficult enough to do simple thinking. Rather, decision-makers need to view their problems in an organized but complex framework that allows for interaction and interdependence among factors and still enable them to think about their problem in a simple way. This new way of thinking should be accessible to all without straining human being's innate capabilities (Saaty, 1990). The AHP provides exactly such a framework. It enables decision-makers to cope with the intuitive, the rational, and the irrational, all at the same time, when they make multicriteria decisions. The decision-makers can use the AHP to integrate their perceptions and purposes into an overall synthesis. There are three principles incorporated in the AHP. They are the principle of constructing hierarchy, the principle of establishing priorities, and the principle of logic consistency (Saaty1980, 1986, 1990). Based on these principles, eight basic steps were condensed into a brief outline for applying AHP by Saaty. The alternative $j$ with the highest overall priority $\left(O P_{j}\right)$ over other alternatives is the best one. For a three-level AHP problem, we have $O P_{j}=\sum_{i=1}^{n} w_{j} w_{i j}$. Where $w_{l}$ is the normalized relative weight of criterion $i ; w_{i j}$ is the relative priority of alternative $j$ against the criterion $i$.

\footnotetext{
" All the notations introduced in this section will be frequently used throughout the paper without any further explanations except they have different meanings.
} 
The main challenges of applying AHP lies in two aspects: how to construct a relevant hierarchy and how to establish the priorities while keeping logic consistency. Although these issues are worthwhile dedicated research, they are beyond the scope of this paper.

\section{AHP-based Approach to Design Evaluation}

A design can be depicted as follows: in the case of $n$ FRs (indexed by i) for an uncoupled design with $m$ design alternatives (indexed by $\mathrm{j}$ ). With respect to the characteristics of the design evaluation in axiomatic design, the hierarchy for selecting the best design is built up as shown in Fig.1.

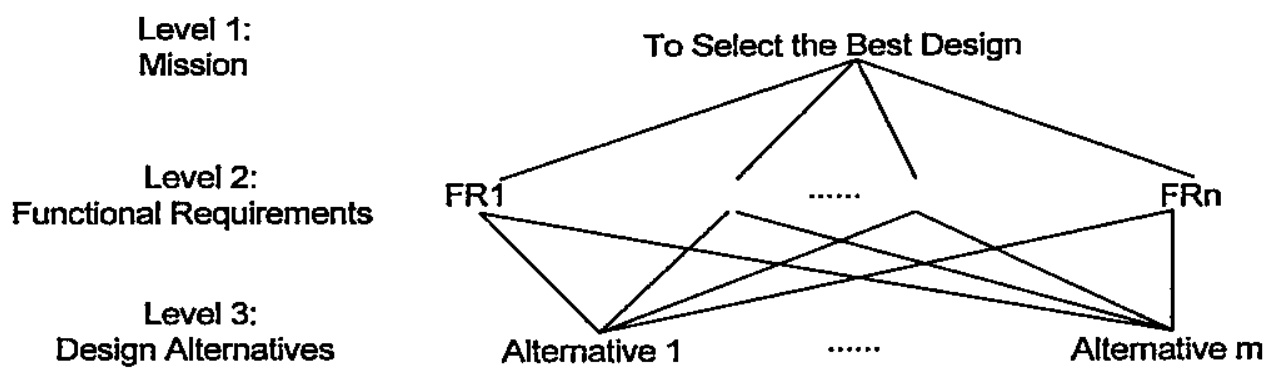

Figure 1. Hierarchy for Selecting the Best Design

With the hierarchy being constructed, the next critical step is to defining the $w_{j}$. Usually, pairwise comparisons are necessary to calculate $w_{i j}$. With given probabilities of DPs successfully satisfying FRs, however, a direct way of defining $w_{i j}$ is adopted in this paper. Based on the conventions of AHP, two principles as follows must be stuck to when defining $w_{i j}$. 1) When comparing any two alternatives, the decision-maker never judges one to be infinitely better than another under any criterion. This means $w_{i j}$ can be neither 0 nor the positive infinite. 2) The logic consistency must be maintained. In summary, $w_{i j}$ must satisfy:

a) $W_{i j} \in[\varepsilon, K], \mathrm{K}$ is a postive real number, $\varepsilon$ is a small but positive real number;

b) $w_{i a}>w_{i b}$ if $\mathrm{p}_{\mathrm{ia}}>p_{i b} ; w_{l a}=w_{i b}$ if $\mathrm{p}_{\mathrm{ia}}=p_{l b}$;

c) $w_{a} a=w_{a b}$ if $p_{a}=p_{a b}$ for any $\alpha, \beta \in \mathrm{N}, \alpha \neq \beta ; \mathrm{a}, \mathrm{b} \in \mathrm{M}$.

Here $w_{i j}$ is defined as follows.

$$
w_{l j}=K+\log _{2} p_{y j}, \mathrm{~K}=\log _{2} \frac{1}{\min \left\{p_{y} \mid i \in N, j \in M\right\}}+\varepsilon
$$

The overall priority of alternative $\mathrm{j}$ is calculated as

$$
O P_{J}=\sum_{i=1}^{n} w_{l} w_{l}
$$

Where $w_{i}$ is the normalized relative importance of $F R_{i}$, which can be assigned by the designers or decision makers based on the practical interest.

\section{Dealing with Relative Importance of FRs}

With the consideration of the cost, preference of the decision makers, the technology constraints and other matters relevant in the application, often, designers have been facing at the importance issue of FRs when they evaluate design alternatives. Different FRs may have different importance. The relative importance of FRs should impact the evaluation of the design alternatives. For example, suppose alternative $j$ is the best design alternative under a setting of relative importance. When the relative importance change, however, alternative $\mathrm{j}$ may not be the best choice any more. As other multiple criteria decision making 
methodologies, AHP-based design evaluation approach handles this issue by assigning different weighting factors to different criteria. In this section, we try to find the relationship between AHP and IA based approaches in terms of the results derived. A comparison of the ability to deal with the relative importance issue of the two approaches will be conducted by an example as well.

\section{Consistency of AHP with IA: When FRs Are Equally Important}

The consistency of AHP and IA is defined as follows: AHP-based approach can achieve consistent results with which derived by IA-based approach for design evaluation if all FRs are equally important. More specifically, when all FRs are equally important, if $I_{a} \leq I_{b}$ holds, $O P_{a} \geq O P_{b}$ holds for any a, b $\in \mathrm{M}$.

Where, $\mathrm{i} \in \mathrm{N}=\{1,2, \ldots, \mathrm{n}\} ; \mathrm{j} \in \mathrm{M}=\{1,2, \ldots, \mathrm{m}\} ; I_{a}\left(I_{b}\right)$ is the information content of the design alternative $a(b)$ educed from the axiomatic design approach; $O P_{a}\left(O P_{b}\right)$ is the overall priority of the design alternative $a(b)$ derived from the AHP.

Proof

Step 1: Calculate $w_{i}$.

When the IA approach is applied to evaluate the design alternatives, all FRs are treated as equally important. This implies that all FRs in the hierarchy depicted in Fig.1 have an identical weight. By normalizing, we have

$$
w_{i}=\frac{1}{n} \text { for all } i \in N
$$

Step 2: Calculate $w_{l]}$ as defined in (3).

Step 3: Based on Equ. (1), (3) and (4), we can deduce as follows:

$$
\begin{aligned}
& I_{a} \leq I_{b} \Rightarrow \sum_{i=1}^{n} \log _{2} \frac{1}{p_{l a}} \leq \sum_{i=1}^{\pi} \log _{2} \frac{1}{p_{i b}} \Rightarrow \\
& -\sum_{i=1}^{n} \log _{2} p_{1 a} \leq-\sum_{i=1}^{n} \log _{2} p_{1 b} \Rightarrow \sum_{i=1}^{n} \log _{2} p_{1 \mathrm{a}} \geq \sum_{i=1}^{n} \log _{2} p_{1 b} \Rightarrow \\
& n K+\sum_{i=1}^{n} \log _{2} p_{t a} \geq n K+\sum_{|=|}^{n} \log _{2} p_{t b} \Rightarrow \\
& \sum_{l=1}^{n}\left(K+\log _{2} p_{l a}\right) \geq \sum_{i=1}^{n}\left(K+\log _{2} p_{l b}\right) \Rightarrow \sum_{j=1}^{n} w_{t a} \geq \sum_{j=1}^{n} w_{l b} \Rightarrow \frac{1}{n} \sum_{l=1}^{n} w_{l a} \geq \frac{1}{n} \sum_{l=1}^{n} w_{l b} \Rightarrow \\
& \sum_{i=1}^{n}\left(\frac{1}{n} * w_{t a}\right) \geq \sum_{i=1}^{n}\left(\frac{1}{n} * w_{t b}\right) \Rightarrow O P_{a} \geq O P_{b}
\end{aligned}
$$

This implies $I_{a} \leq I_{b} \Rightarrow O P_{a} \geq O P_{b}$. The Theorem 1 holds.

\section{Dealing with Unequal Relative Importance of FRs}

In the axiomatic design, concerning the importance issue of different FRs in design evaluation, as Prof. Suh states in his book:

"The Information Axiom provides a powerful criterion for making such decisions without the use of arbitrary weighting factors used in other decision-making theories. ...the intention of the designer and the importance assigned to each $F R$ by the designer are represented by the design range. If it is a critical FR that must be satisfied within a tight tolerance, the designer would give a narrow range (Suh, 1998)."

There are no weighting factors used to reflect the relative importance of FRs. The design range corresponding to the important FR will be narrowed down. However, the 'narrowing' scheme may not work in some cases. For simplicity, let's illustrate that with an example: two independent FRs $\left(\mathrm{FR}_{1}\right.$ and $\mathrm{FR}_{2}$ ) and two uncoupled design alternatives $a$ and $b$. 
Suppose the following relationships hold:

1) $I_{a}>I_{b}$, or equivalently, $p_{1 a} p_{2 a}<p_{1 b} p_{2 b}$ where $p_{1 a}<p_{1 b}$ but $p_{2 a}>p_{2 b}$.

2) The design range for $\mathrm{FR}_{2}$ and the system ranges of design alternatives $a$ and $b$ have an identical starting point, the point $\mathrm{A}$, as demonstrated in Fig. 2. Moreover, $C R_{2 \mathrm{a}}=C R_{2 b}<S R_{2 a}<S R_{2 b}$.



Figure 2 Design range for FR2 and system ranges for design aiternatives

The above conditions imply that, the design alternative $a$ has a better satisfaction of FR2 than $b$, although the alternative $a$ is worse than $b$ in terms of the overall satisfactions of both FR1 and FR2.

Based on the Equ. (1) and (2), we have

$$
\begin{aligned}
& I_{a}=\log _{2} \frac{1}{p_{1 a}}+\log _{2} \frac{1}{p_{2 a}}=\log _{2} \frac{1}{p_{1 a}}+\log _{2} \frac{S R_{2 a}}{C R_{2 n}} \\
& I_{b}=\log _{2} \frac{1}{p_{1 b}}+\log _{2} \frac{1}{p_{2 b}}=\log _{2} \frac{1}{p_{1 b}}+\log _{2} \frac{S R_{2 b}}{C R_{2 b}}
\end{aligned}
$$

Now, suppose we know $F R_{2}$ is important than $F R_{1}$. Based on the Information Axiom approach, the $\mathrm{CR}_{2}$ will be narrowed to $\delta \mathrm{CR}_{2}(0<\delta<1)$. Surely, the more significant the importance is, the smaller the $\delta$.

By narrowing the $\mathrm{DR}_{2}$ to $\delta \mathrm{DR}_{2}$, we have $C R_{2 a}{ }^{\prime}=C R_{2 b}{ }^{\prime}=\delta D R_{2}=\delta C R_{2 a}=\delta C R_{2 b}$, therefore,

$$
\begin{aligned}
& I_{a}^{\prime}=\log _{2} \frac{1}{P_{t a}}+\log _{2} \frac{S R_{2 a}}{\delta \cdot C R_{2 a}}=I_{a}+\log _{2} \frac{1}{\delta} \\
& I_{b}^{\prime}=\log _{2} \frac{1}{p_{1 b}}+\log _{2} \frac{S R_{2 b}}{\delta \cdot C R_{2 b}}=I_{b}+\log _{2} \frac{1}{\delta}
\end{aligned}
$$

Hence, $I_{a}^{\prime}>I_{b}^{\prime}$ since $I_{a}>I_{b}$. This means the design alternatives $b$ is always better than alternative $a$, no matter to any extent the design range of FR2 is narrowed. In other words, the alternative $a$, which has a better satisfaction of FR2, can never become better than alternative $b$, no matter how important the FR2 becomes. This conclusion is against the common sense. Supporting by this counter example, one could conclude that narrowing down the design range could not correctly reflect the relative importance of the FRs in some cases.

Let's see how AHP-based approach deals with the same problem. Suppose originally, $\mathrm{FR}_{1}$ and $\mathrm{FR}_{2}$ are treated equally important and $b$ is better than $a\left(I_{a}>I_{b}\right)$. According to Theorem 1, $O P_{a}<O P_{b}$ and $w_{1}=w_{2}=0.5$ hold. It is expected that the alternative a will become better than $b$ if $\mathrm{FR}_{2}$ is more important than $\mathrm{FR}_{1}$. This means we can find a certain pair of $W_{1}^{\prime}$ and $W_{2}^{\prime}$ where $W_{2}{ }^{\prime}>w_{1}{ }^{1}$, so that $O P_{a}>O P_{b}$ holds.

With the given conditions, $p_{1 a} p_{2 a}<p_{1 b} p_{2 b}, p_{l a}<p_{1 b}$ but $p_{2 a}>p_{2 b}$ we can derive that, 
$\log _{2} p_{1 a}-\log _{2} p_{1 b}-\log _{2} p_{2 a}+\log _{2} p_{2 b}<0$

Suppose $O P_{a}^{\prime}>O P_{b}^{\prime}$ holds, i.e.,

$w_{1}^{\prime} *\left(K+\log _{2} p_{1 a}\right)+w_{2}^{\prime}\left(K+\log _{2} p_{2 a}\right)>w_{1}^{\prime} *\left(K+\log _{2} p_{1 b}\right)+w_{2}^{\prime} *\left(K+\log _{2} p_{2 b}\right)$

Solve the above inequality, with considering $w_{1}^{\prime}+w_{2}^{\prime}=1$, we have

$0<w_{1}<\frac{\log _{2} p_{2 b}-\log _{2} p_{2 a}}{\log _{2} p_{1 a}-\log _{2} p_{2 a}+\log _{2} p_{2 b}-\log _{2} p_{1 b}}$

$w_{2}^{\prime}>\frac{\log _{2} p_{1 a}-\log _{2} p_{1 b}}{\log _{2} p_{1 a}-\log _{2} p_{2 a}+\log _{2} p_{2 b}-\log _{2} p_{1 b}}$

We can further prove that $1>W_{2}{ }^{\prime}>0.5>W_{1}{ }^{\prime}>0$. This means that we can find $W_{1}^{\prime}$ and $W_{2}^{\prime}$ so that alternative $a$ will become better than alternative $b$ as long as $\mathrm{FR}_{2}$ becomes as important as such that the inequality (8) holds. Obviously, this conclusion is consistent with which is expected if AHP is applied.

\section{Conclusion}

We conclude with general remarks about the use of IA and AHP for design evaluation in axiomatic design.

1) General speaking, AHP can be a very pertinent method for design evaluation in axiomatic design. It can even be used in some situations where the IA is difficult to deal with, for example, the situation with incomplete and/or fuzzy information.

2) The measurement used in IA approach-the Information Content-has a clear physical meaning. It is the joint probability of all FRs being satisfied simultaneously. The measurement used in AHP-the overall priority, however, does not imply a precise physical meaning.

3) When all FRs are equally important and all probabilities of success of satisfying FRs are known, either IA approach or AHP can be employed to do design alternative evaluation.

4) When FRs are not equally important, AHP instead of IA approach is suggested to do design evaluation.

\section{Reference}

Jiao, J.X. and Tseng, M.M. (1998), "Fuzzy ranking for concept evaluation in configuration design for mass customization", Concurrent Engineering: Research and Applications, 6(3), 189-206.

Saaty, T. L. (1980), The Analytic Hierarchy Process, New York: McGraw-Hill.

Saaty, T. L. (1986), "Axiomatic foundations of the analytic hierarchy process", Management Science, 32 (7), 841-855.

Saaty, T. L. (1990), Decision Making for Leaders: the Analytic Hierarchy Process for Decisions in A Complex World ( $2^{\text {nd }}$ edition), Pittsburgh: RWS Publications.

Suh, N. P. (1990), The Principles of Design, New York: Oxford University Press.

Suh, N. P. (1998), The Principles of Design ( ${ }^{\text {nd }}$ edition), In processing.

Vargas, L. G. \& Zahedi, F. (Eds.) (1993), Preface. Journal of Mathematical Computing and Modeling, 17(4/5), pix-xi. 